\title{
Comparison of Hostility and Sleep Disorders among Nursing Students with Post-Traumatic Stress Disorder
}

\author{
Borzoo Amirpour ${ }^{*}$, Mehdi Merati ${ }^{2}$ \\ ${ }^{1}$ Department of Psychology, Payam-e-Noor University, Tehran, Iran \\ 'Department of Clinical Psychology, Sari Branch, Islamic Azad University, Sari, Iran
}

\section{ABSTRACT}

Introduction: Both at the $11^{\text {th }}$ edition of the international classification of diseases (ICD-11) and in the Fifth edition of the diagnostic and statistical manual of mental disorders (Fifth editionDSM-5), It has been noted that the attitudes (toward self, others, world and future) and quality of sleep can be seriously impaired by post-traumatic stress disorder (PTSD). The present study was conducted to compare hostility and sleep disorders among nursing students with and without PTSD. Materials and Methods: This descriptive-comparative causal study was performed on 190 (140 females \& 50 males) nursing students from Islamic Azad University, Toyserkan, Iran. Participants were chosen by stratified sampling. Three questionnaires were used in this study, including the Post-Traumatic Stress Disorder Checklist-Civilian version (PCL-C), hostility subscale from Buss \& Perry aggression questionnaire, and sleep disorder subscale from Mississippi scale for combat-related PTSD. Results: Fifty-six out of the 190 students were identified with PTSD symptoms. This group of students had significantly higher scores on hostility and sleep disorders. Regression analysis indicated that sleep disorders and hostility were strong positive predictors for PTSD. Conclusion: Due to considerable prevalence and impact of PTSD on sleep quality and hostility in patients who suffering from PTSD, intervention to promote appropriate diagnosis and treatment seems to be crucial.

* Corresponding Author: Borzoo Amirpour

E-mail: borzooamirpour@gmail.com 


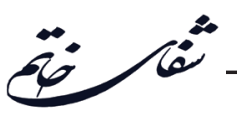

مقايسهٔ خصومت و اختلالات خواب در ميان دانشجويان رشتهُ يرستارى با اختلال استرس يس از سانحه

\author{
برزو امير يور ":، مهدى مر آتى ' \\ 'كروه روانشناسى، دانشًاه ييام نور، تيران، ايران \\ كَّروه روانشناسى بالينى، واحد سارى، دانشًاه آزاد اسلامى، سارى، ايران
}

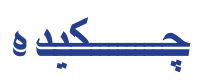

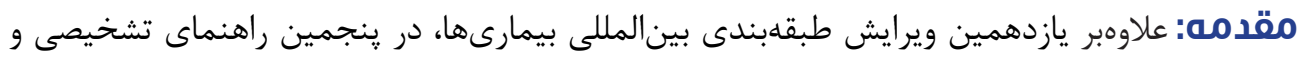

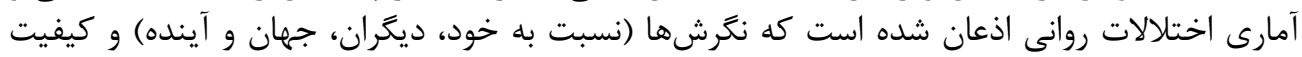

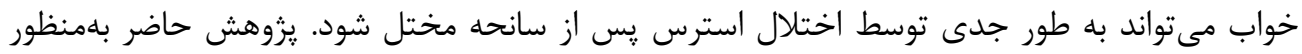

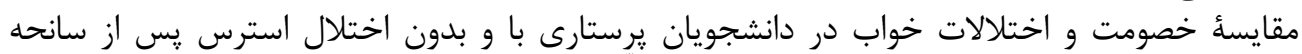

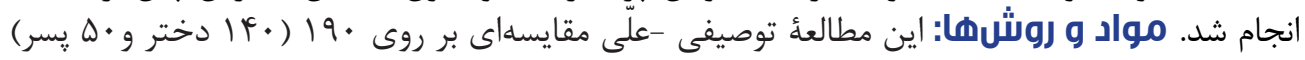

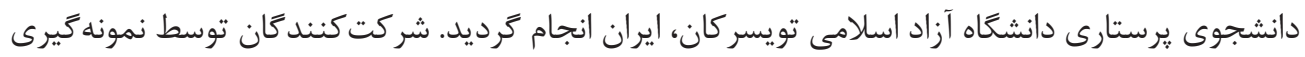

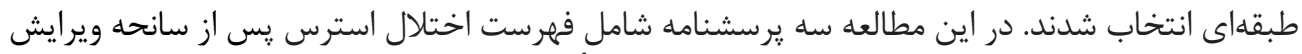

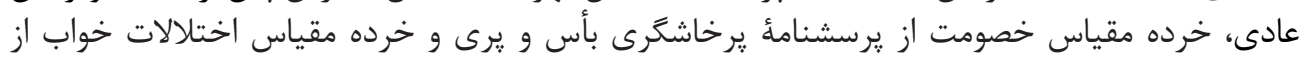

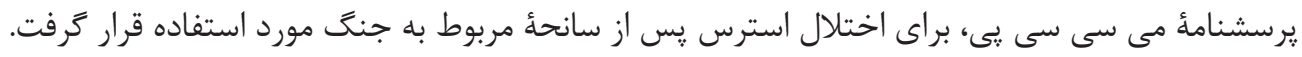

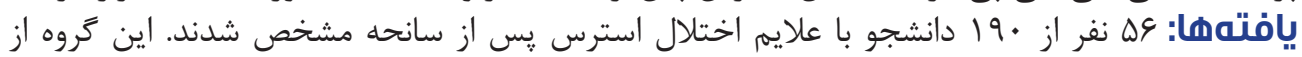

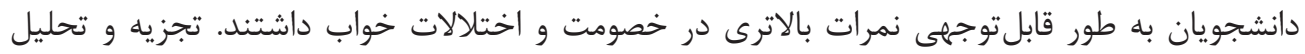

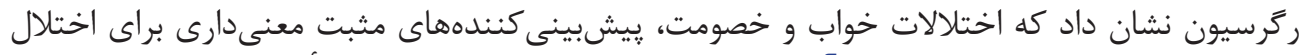

كليد وازهها:

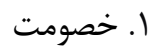

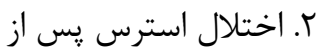
سانحه

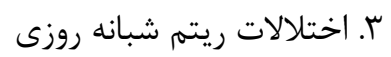
خواب

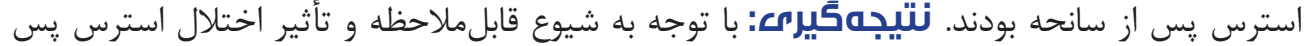

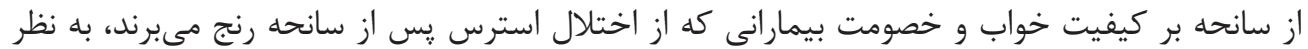
مىرسد كه مداخله براى بيشرفت تشخيص و و درمان بسيار مههم است. 
مخاطره انداختن بهبودى نيز تداخل ايجاد مى كنند. خواب براى

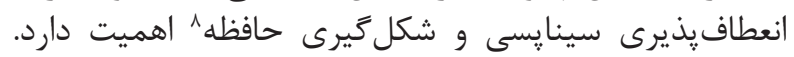

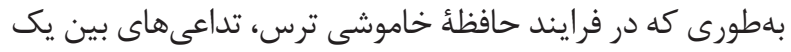

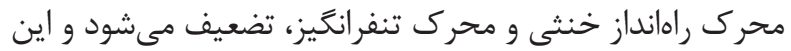

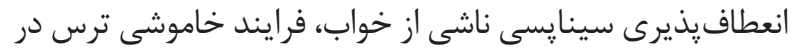

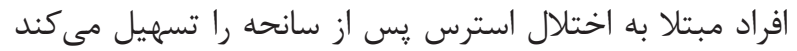

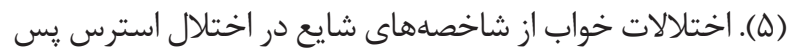

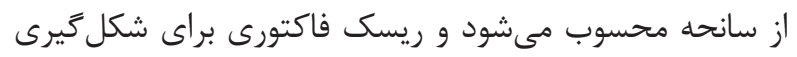
اين اختلال به دنبال مواجهه با تروما تلقى مىشئ مشوند.

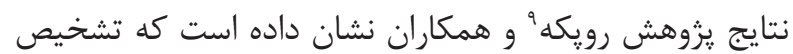

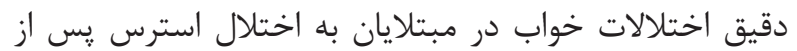

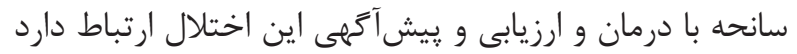

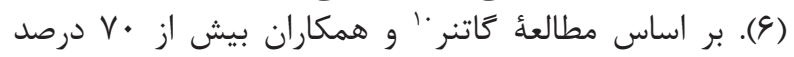

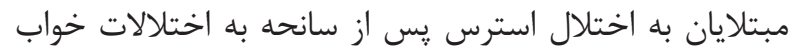

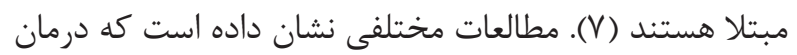

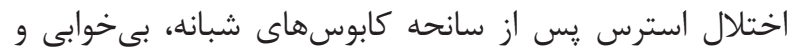

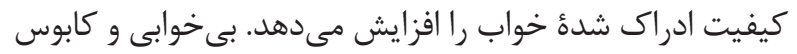

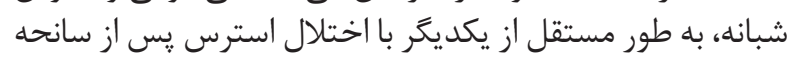

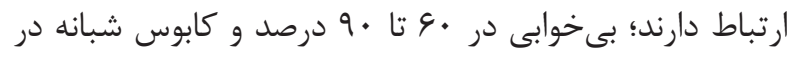

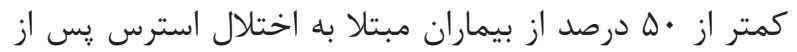

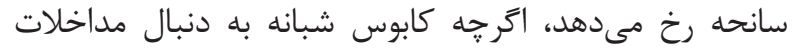

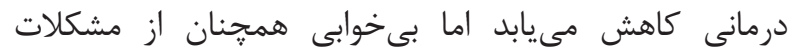
باقيمانده و رايج اين اختلال قلمداد مىشود (^).

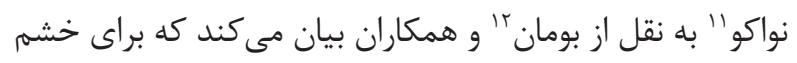

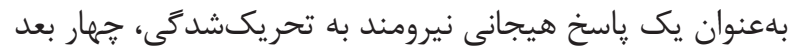

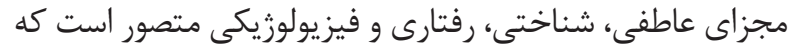

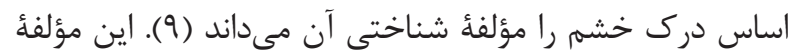

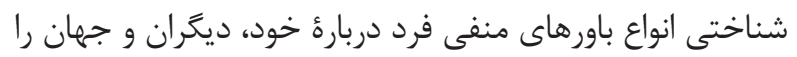

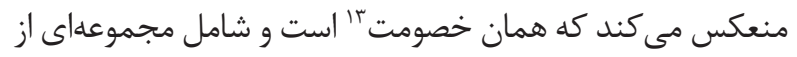

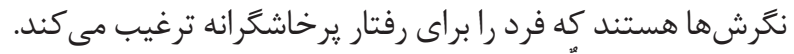

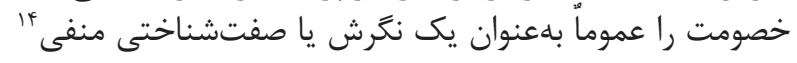

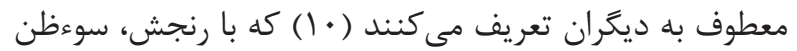

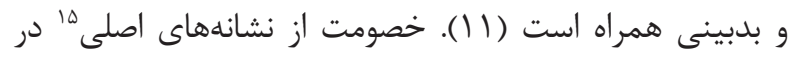

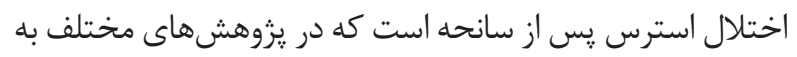

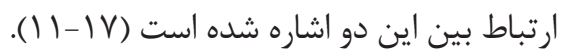

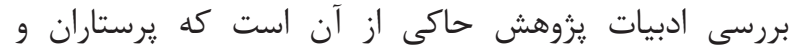

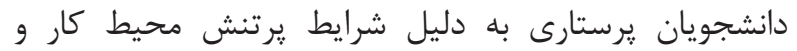

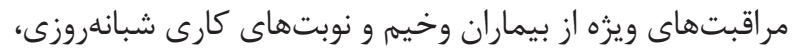

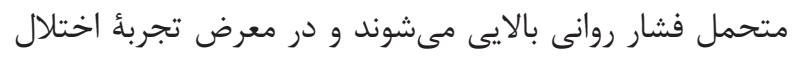

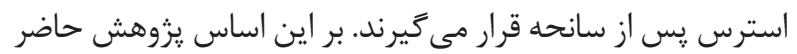

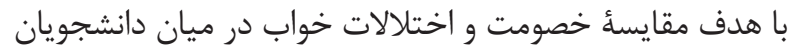

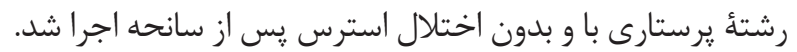

\section{${ }^{1}$ Post-traumatic stress disorder}

${ }^{2}$ Traumatic

${ }^{3}$ World health organization

${ }^{4}$ International classification of diseases, 11 th revision, clinical modification

${ }^{5}$ Complex post traumatic stress disorder

${ }^{6}$ Clinical utility

${ }^{7}$ Fear extinction memory

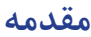

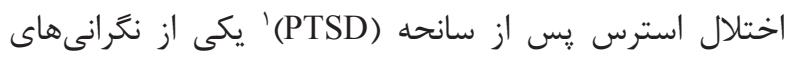

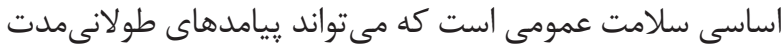

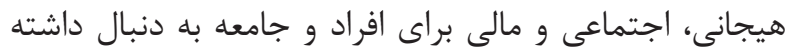

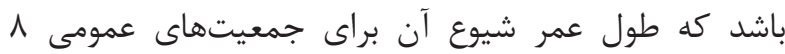

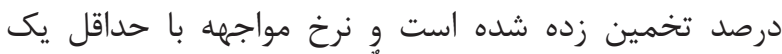

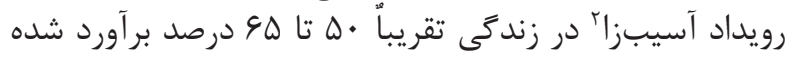

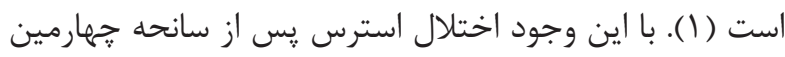

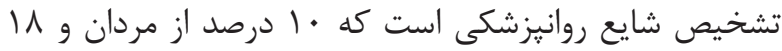

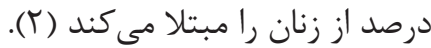

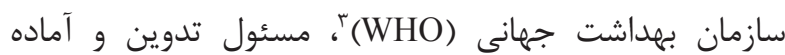

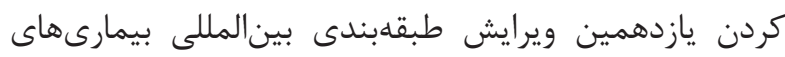
روانى (ICD-11"

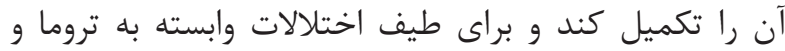

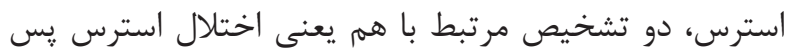

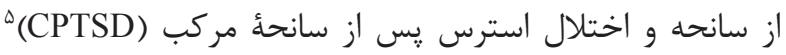

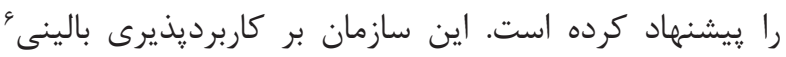

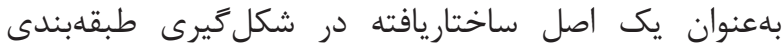

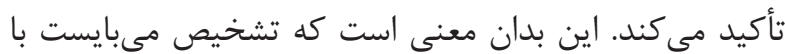

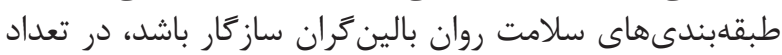

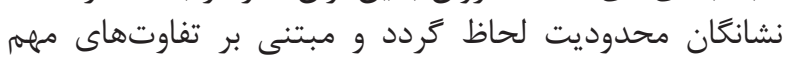

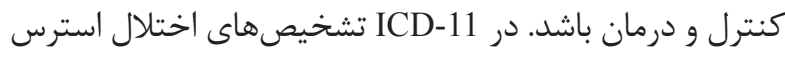

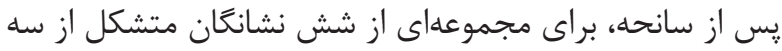

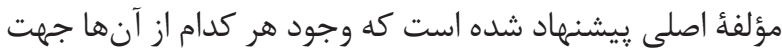

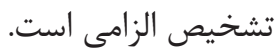

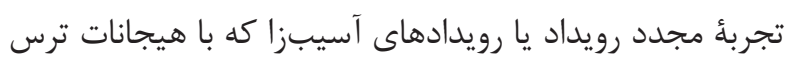

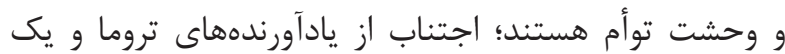

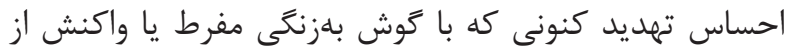

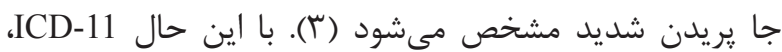

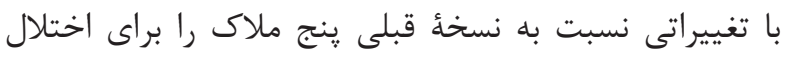

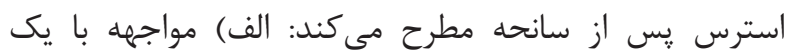

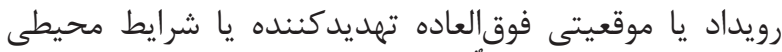

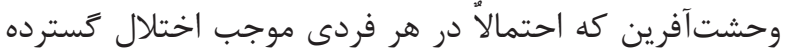

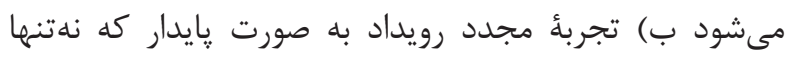

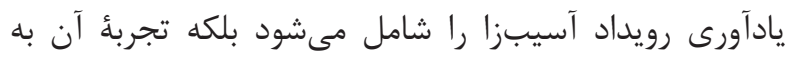

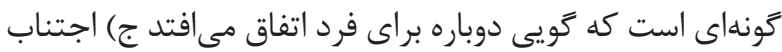

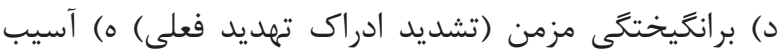
كاركردى قابلملاحظه از نظر بالينى (\$). (1).

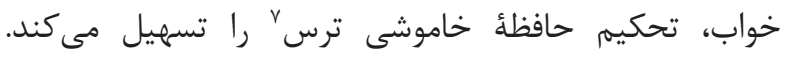

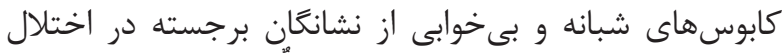

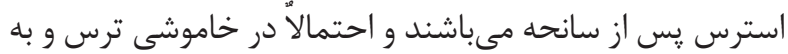

\footnotetext{
${ }^{8}$ Synaptic plasticity and memory formation

${ }^{9}$ Roepke

${ }^{10}$ Gutner

${ }^{11}$ Novaco

${ }^{12}$ Boman

${ }^{13}$ Hostility

${ }^{14}$ Negative attitude or cognitive trait

${ }^{15}$ Hallmark
} 


\section{خرده مقياس خصومت از مقياس يرخاشگرى بأس و يرى}

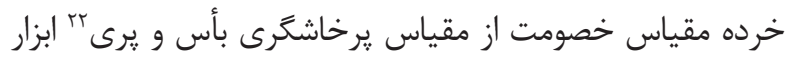

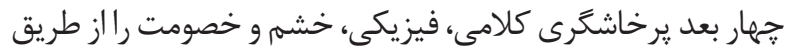

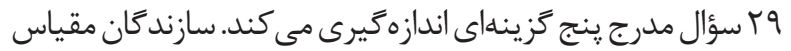

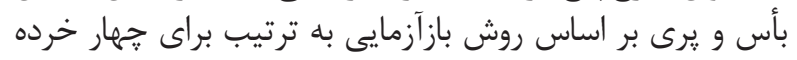

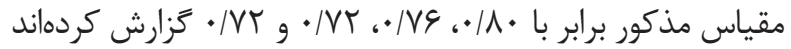
(YY)

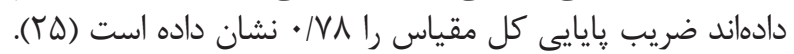

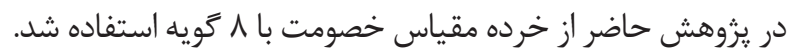

$$
\text { تجزيه و تحليل دادهها }
$$

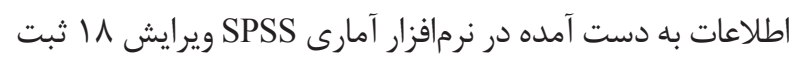

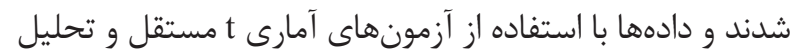

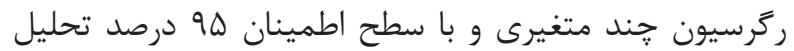

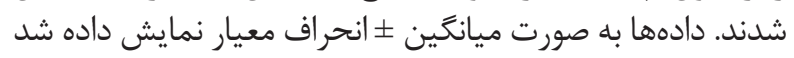

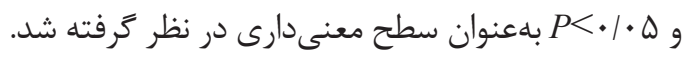

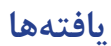

از مجموع •19 يرستار حاضر در يزوهش 19 نفر نمر نمرات بالاتر

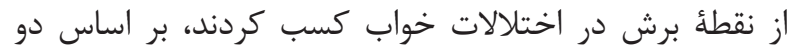

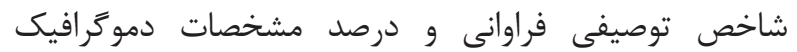

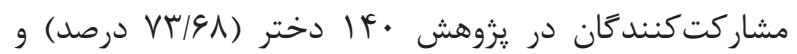

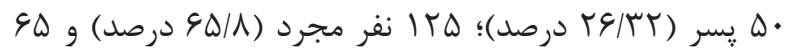

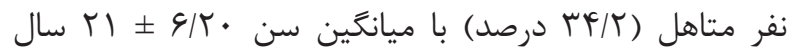

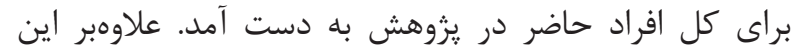

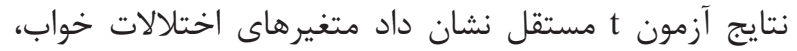

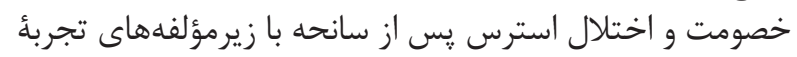

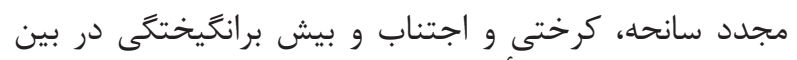

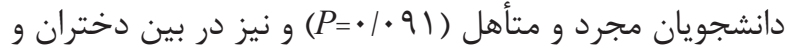

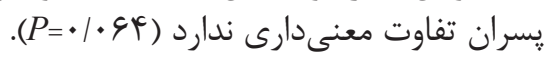

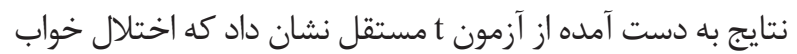

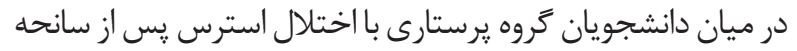

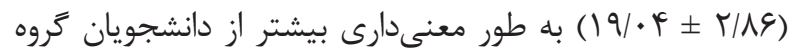

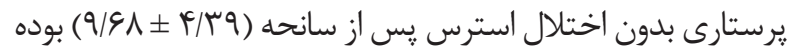

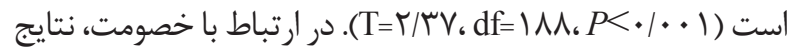

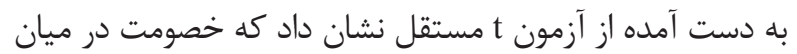

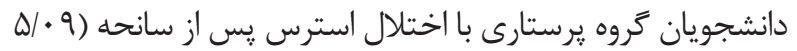

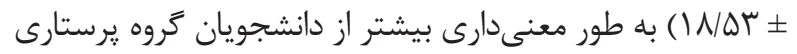

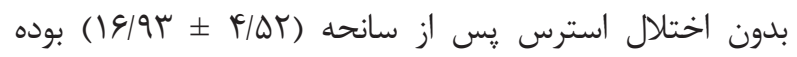

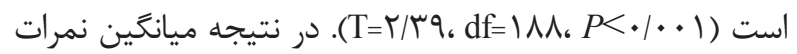

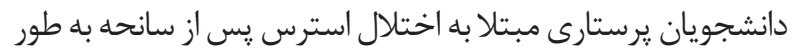

معنى دارى بالاتر از دانشجويان فاقد اين اختلال است (نمودار ().

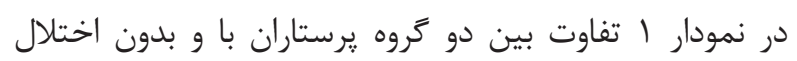

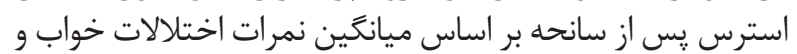
خصومت ارائه شده است.

${ }^{16}$ The post-traumatic stress disorder checklist-civilian version

${ }^{17}$ Weathers

${ }^{18}$ Mississipi scale for post-traumatic stress disorder

${ }^{19}$ Boks

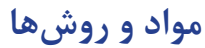

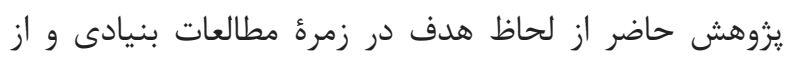

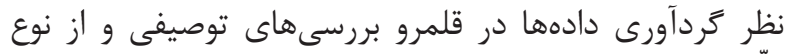

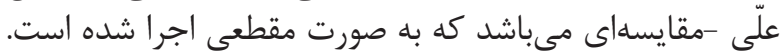

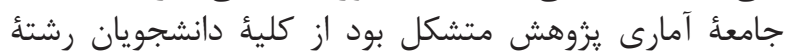

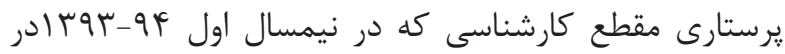

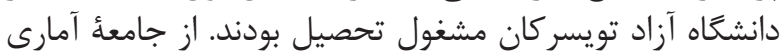

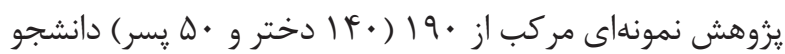

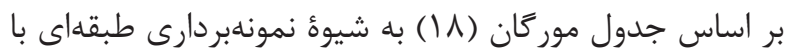

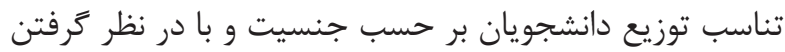

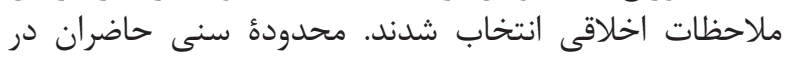

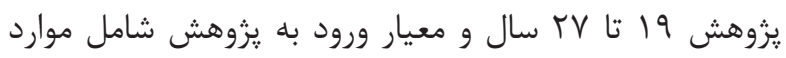

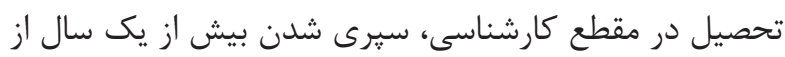

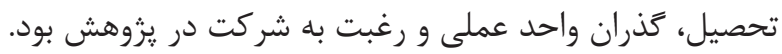

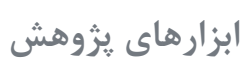

קك ليست اختلال استرس يس از سانحه -نسخهُ عادى

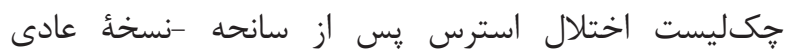
(PCL-C)

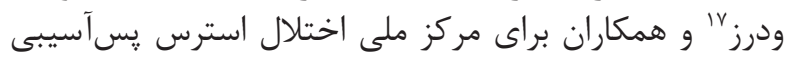

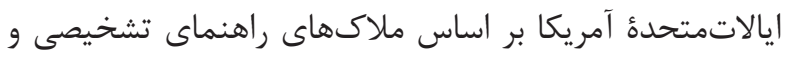

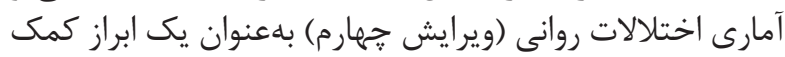

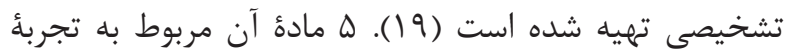

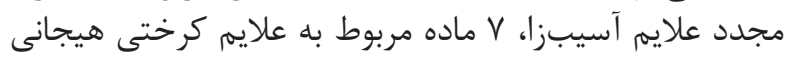

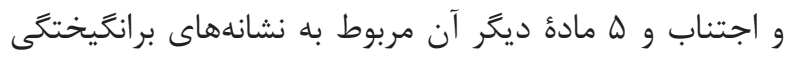

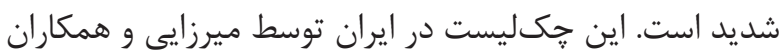

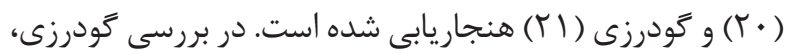

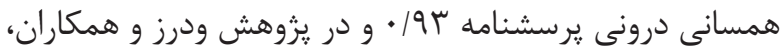

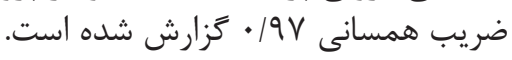

مقياس مى سى سى بِى براى سنجش اختلال استرس

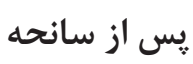

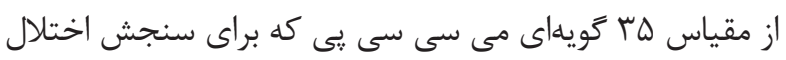

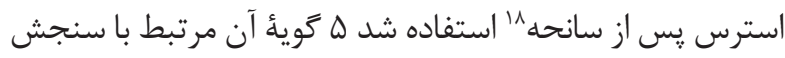

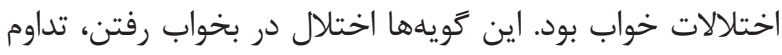

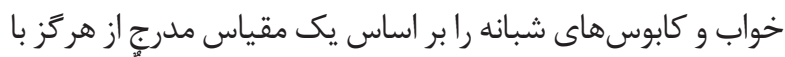

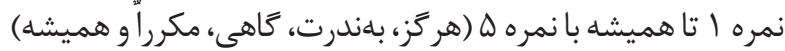

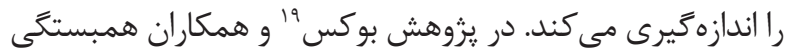

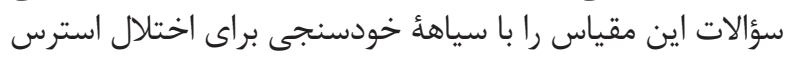

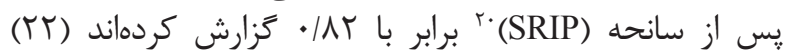

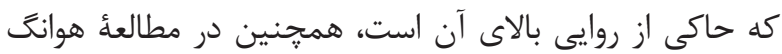

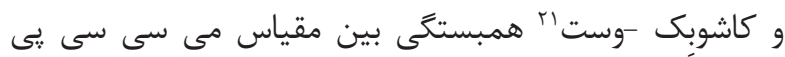

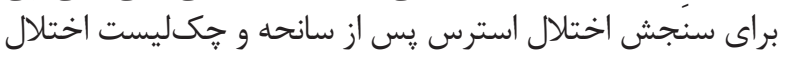

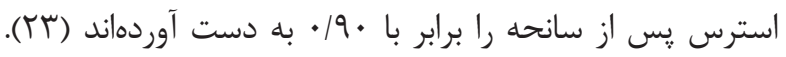

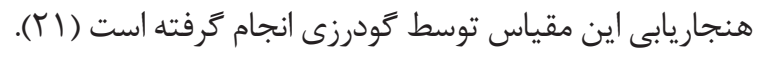

${ }^{20}$ Self-rating inventory for PTSD

${ }^{21}$ Huang and Kashubeck-West

${ }^{22}$ Hostility subscale from Buss and Perry aggression questionnaire 


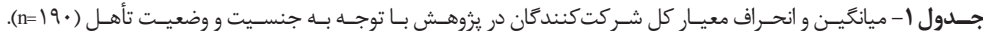

\begin{tabular}{|c|c|c|}
\hline ميانكين 土 انحر اف معيار & متغير يُش بين & متغير ملاك \\
\hline 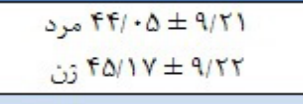 & جنسيت & \multirow{3}{*}{ اختلال استرس هيس از سانحه } \\
\hline 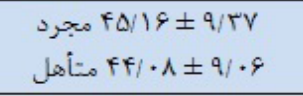 & وضعيت تأهل & \\
\hline$f F / f r \pm q / \Delta F$ & 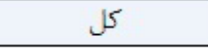 & \\
\hline 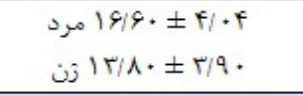 & جنسيت & \multirow{3}{*}{ تجربةً مجدد سانحه } \\
\hline 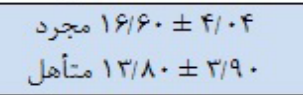 & وضعيت تأهل & \\
\hline $\mid f / r r \pm f / \cdots$ & كل & \\
\hline 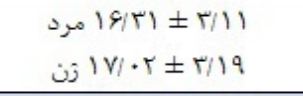 & جنسيت & \multirow{3}{*}{ كرختى و اجتناب } \\
\hline 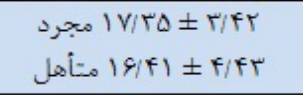 & وضعيت تأهل & \\
\hline $\mathrm{IV/} / \mathrm{r} \pm \mathrm{f} / \mathrm{Af}$ & كل & \\
\hline 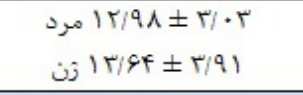 & جنسيت & \multirow{3}{*}{ بيش برانكيختـفى } \\
\hline 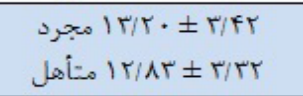 & وضعيت تأهل & \\
\hline $\mid r / \cdot V \pm r / r \Lambda$ & كل & \\
\hline 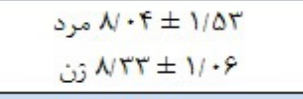 & جنسيت & \multirow{3}{*}{ اختلال خواب } \\
\hline 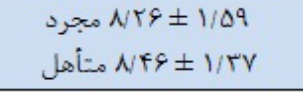 & وضعيت تأهل & \\
\hline$N / T \pm 1 / 01$ & كل & \\
\hline 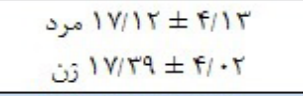 & جنسيت & \multirow{3}{*}{ خصومت } \\
\hline 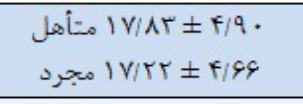 & وضعيت تأهل & \\
\hline$\Leftrightarrow / V / f r \pm f / V F$ & 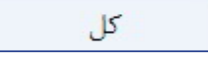 & \\
\hline
\end{tabular}

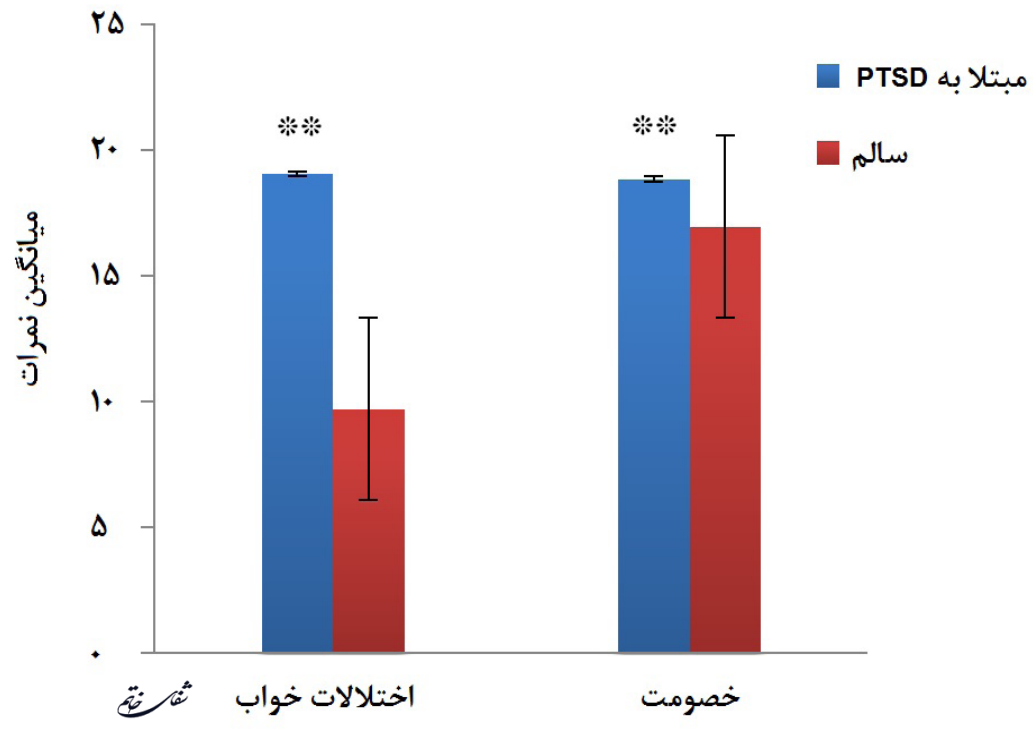

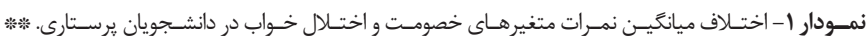

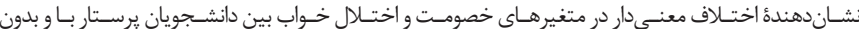

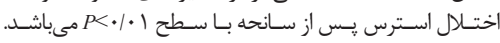


جدول Y-نتايج تحليل ركرسيون جهت ييشبينى اختلال استرس يس از سانحه بر اساس متغيرهاى ييشبين.

\begin{tabular}{|c|c|c|c|}
\hline \multirow{2}{*}{$\begin{array}{l}\text { P value } \\
\text { (sig) }\end{array}$} & \multirow{2}{*}{ مقدار T } & ضرايب استانسارد & \multirow{2}{*}{ متغيرهاى ييش بين } \\
\hline & & Beta & \\
\hline$\cdot 1 \cdots$ & $N|S|$ & - & مقدار ثابت \\
\hline$\cdot 1 \cdot r$ & $4 / 19$ &.$/ T H V$ & اختلالات خواب \\
\hline 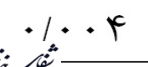 & $r / 9 \Delta$ &.$/ 411$ & خصومت \\
\hline
\end{tabular}

زمينهای ايجاد اختلال استرس يس از سانحه در مطالعات

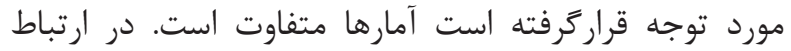

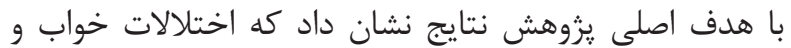

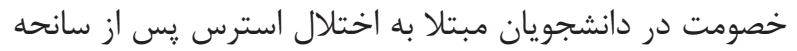

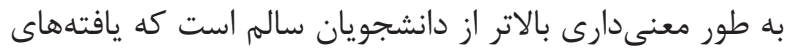

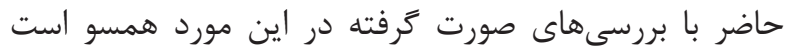

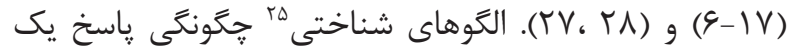

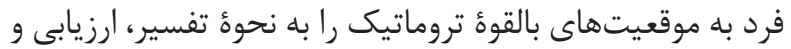

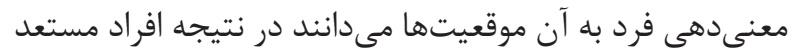

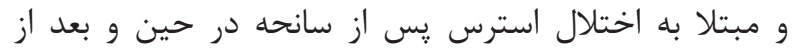

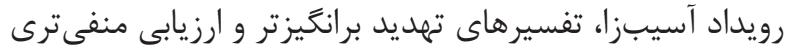

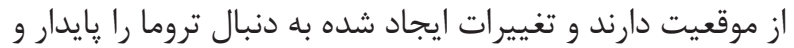

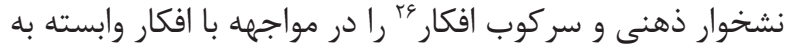

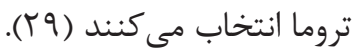

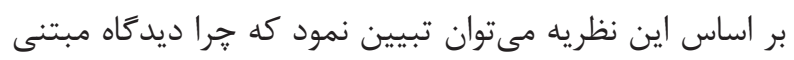

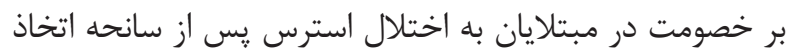

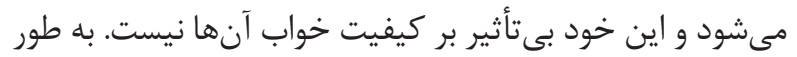

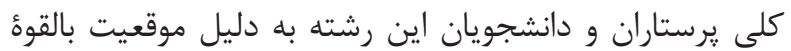

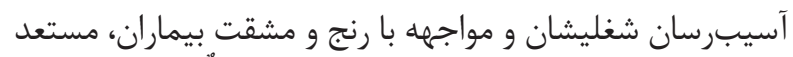

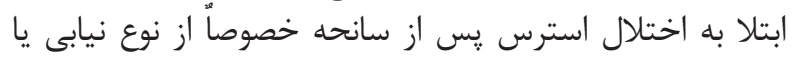

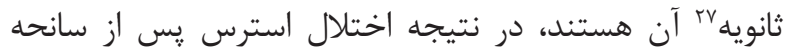

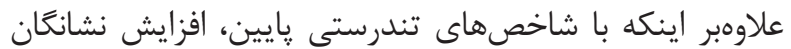

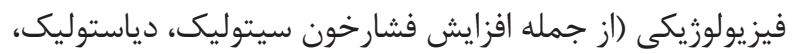

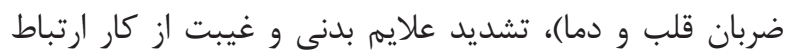

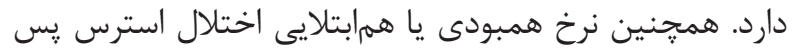

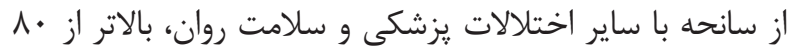

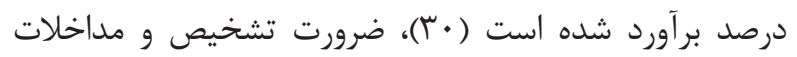

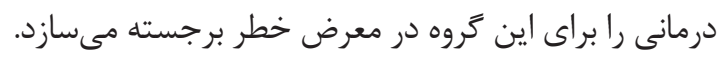
بررسى حاضر با محدوديتهايى همراه بوده است كه به به

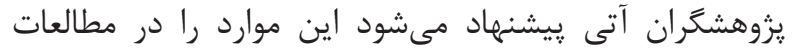

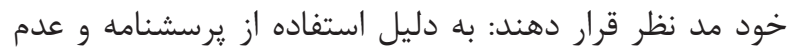

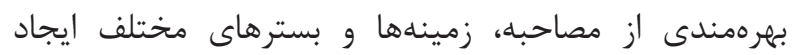

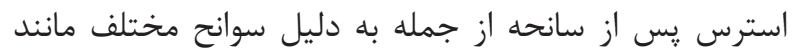

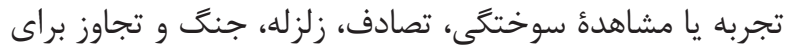

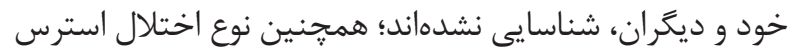

${ }^{23}$ Enter method

${ }^{24}$ Diagnostic and statistical manual of mental disorders, $5^{\text {th }}$

${ }^{25}$ Cognitive models

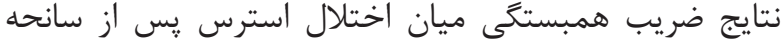

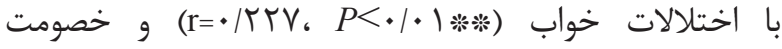
(r=|•r) در كروه مبتلايان به اختلال استرس

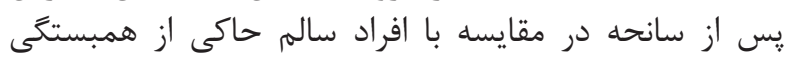

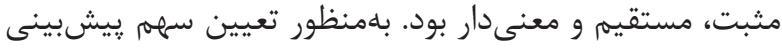

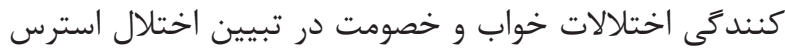

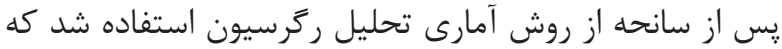

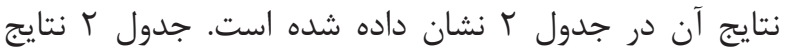

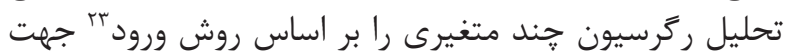

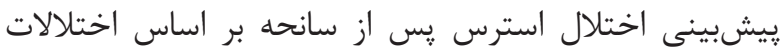
خواب و خصومت نمايش مى ندهد.

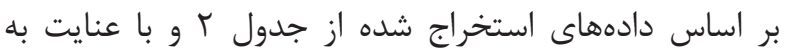

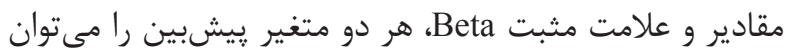

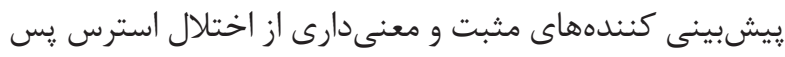

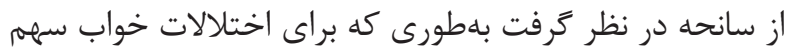

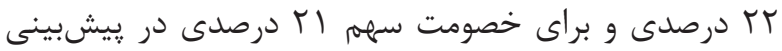

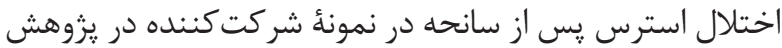
را مىتوان تصور كرد.

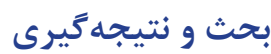

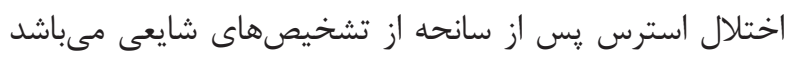

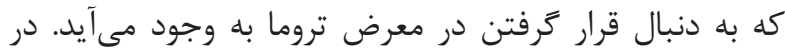

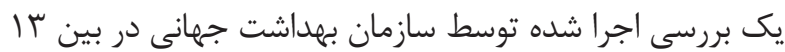

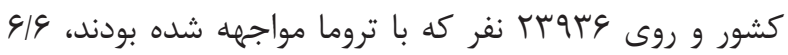

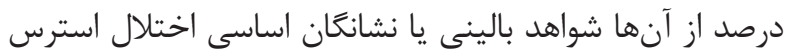

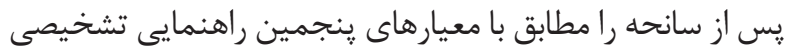

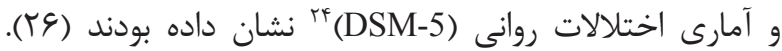

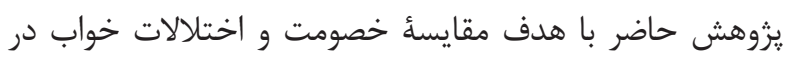

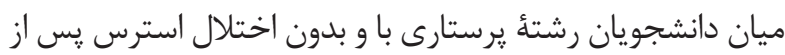

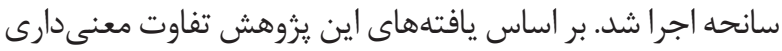

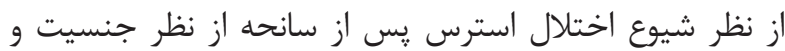

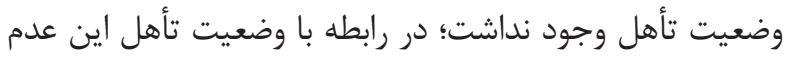

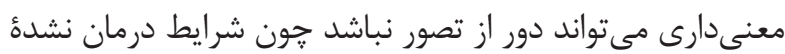

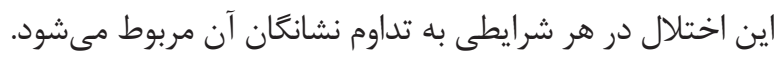
از نظر جنسيتى بسته به جامعأ آمارى يزوهش و اينكه كدام

${ }^{26}$ Rumination about trauma and thought suppression

${ }^{27}$ Secondary traumatic stress 


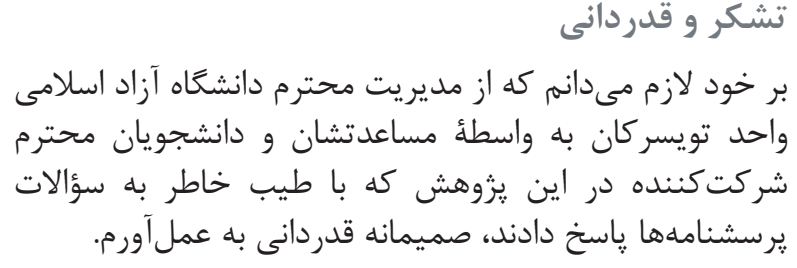

1. Sheldon T. Psychological intervention including emotional freedom techniques for an adult with motor vehicle accident related posttraumatic stress disorder: A case study. Curr Res Psychol. 2014; 5(1): 40-63.

2. Nagpal ML, Gleichauf K, Ginsberg JP. Meta-analysis of heart rate variability as a psychophysiological indicator of posttraumatic stress disorder. J Trauma Treat. $2013 ; 182$ (3): 8-31.

3. Cloitre M, Garvert DW, Brewin CR, Bryant RA, Maercker A. Evidence for proposed ICD-11 PTSD and complex PTSD: a latent profile analysis. Eur J Psychotraumatol. 2013; 15(4): 1-12.

4. Stein DJ, McLaughlin KA, Koenen KC, Atwoli L, Friedman MJ, Hill ED, et al. DSM-5 and ICD-11 definitions of posttraumatic stress disorder: investigating "narrow" and "broad" approaches. Depress Anxiety. 2014; 31(6): 494-505.

5. Van Liempt S. Sleep disturbances and PTSD: a perpetual circle? Eur J Psychotraumatol. 2012; 3. doi: 10.3402/ejpt.v3i0.19142.

6. Roepke S, Hansen ML, Peter A, Merkl A, Palafox C, Danker-Hopfe H. Nightmares that mislead to diagnosis of reactivation of PTSD. Eur J Psychotraumatol. 2013; 4(5): 1-6.

7. Gutner CA, Casement MD, Gilbert KS, Resick PA. Change in sleep symptoms across Cognitive Processing Therapy and Prolonged Exposure: A longitudinal perspective. Behav Res Ther. 2013; 51(12): 817-22.

8. Pigeon WR, Campbell CE, Possemato K, Ouimette P. Longitudinal relationships of insomnia, nightmares, and PTSD severity in recent combat veterans. J Psychosom Res. 2013; 75(6): 546-50.

9. Boman P, Mergler A, Furlong M, Caltabiano N. Anger in Australian indigenous and non-indigenous students. Int Educ J. 2014; 13(2): 15-26.

10. Suls J. Anger and the heart: perspectives on cardiac risk, mechanisms and interventions. Prog Cardiovasc Dis. 2013; 55(6): 538-47.

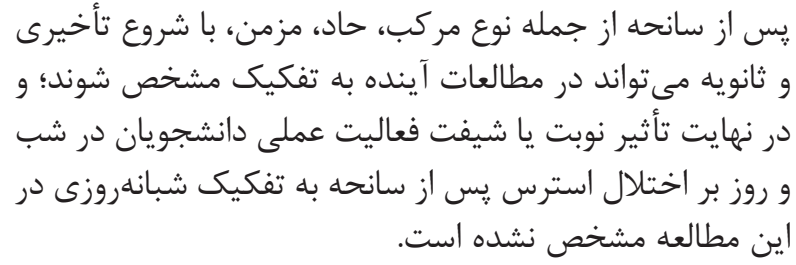

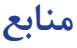

11. Teten AL, Miller LA, Stanford MS, Petersen NJ, Bailey SD, Collins RL, et al. Characterizing aggression and its association to anger and hostility among male veterans with post-traumatic stress disorder. Mil Med. 2010; 175(6): 405-10.

12. Beckham JC, Flood AM, Dennis MF, Calhoun PS. Ambulatory cardiovascular activity and hostility ratings in women with chronic posttraumatic stress disorder. Biol Psychiatry. 2009; 65(3): 268-72.

13. Vrana SR, Hughes JW, Dennis MF, Calhoun PS, Beckham JC. Effects of posttraumatic stress disorder status and covert hostility on cardiovascular responses to relived anger in women with and without PTSD. Biol Psychol. 2009; 82(3): 274-80.

14. Birkley EL, Eckhardt CI. Anger, hostility, internalizing negative emotions, and intimate partner violence perpetration: A meta-analytic review. Clin Psychol Rev. 2015; 37(2): 40-56.

15. Elbogen EB, Wagner HR, Fuller SR, Calhoun PS, Kinneer PM. Correlates of anger and hostility in Iraq and Afghanistan war veterans. Am J Psychiatry. 2010; 167(9): 1051-8.

16. Galovski TE, Elwood LS, Blain LM, Resick PA. Changes in anger in relationship to responsivity to PTSD Treatment. Psychol Trauma. 2014; 6(1): 56-64.

17. Kathleen KT. The psychoneuroimmunology of chronic disease: exploring the links between inflammation, stress, and illness. American Psychological Association. 2010.

18. Nadri E, Seife Naraghi M. Research methods and how to evaluate it in humanities sciences with emphasis on educational science $5^{\text {th }}$ ed. Tehran: Arasbaran Press. 2012.

19. Weathers FW, Litz BT, Herman DS, Huska JA, Keane T. The PTSD checklist (PCL). reliability, validity and diagnostic utility. San Antonio; $9^{\text {th }}$ Annual Meeting of International Society for Traumatic Stress Hisser Studies. 1993.

20. Mirzaee J. Karami GR, Ameli J, Hemmati MA. 
Investigation of clinical diagnosis by psychological tests in PTSD outpatients and inpatients. J Mil Med. 2004; 6 (3): 201-8.

21. Goodarzi MA. Reliability and validity of posttraumatic stress disorder Mississippi scale. J psychol. 2003; 7(2): 135-78.

22. Boks MP, Van Mierlo HC, Rutten BP, Radstake TR, De Witte L, Geuze E, et al. Longitudinal changes of telomere length and epigenetic age related to traumatic stress and post-traumatic stress disorder. Psychoneuroendocrinology. 2015; 51: 506-12.

23. Huang HH, Kashubeck-West S. Exposure, agency, perceived threat, and guilt as predictors of posttraumatic stress disorder in veterans. J Couns Dev. 2015; 93(1): 3-13.

24. Buss AH, Perry M. The aggression questionnaire. J Pers Soc Psychol. 1992; 63(3): 452-9.

25. Samani S. Study of reliability and validity of buss and Perry's aggression questionnaire. Iran J Psychiat Clin Psychol. 2008; 13(4): 359-65.

26. Williamson JB, Porges EC, Lamb DG, Porges
SW. Maladaptive autonomic regulation in PTSD accelerates physiological aging. Front Psychol. 2015; 5(571): 1-12.

27. Morin CM, Belleville G, Bélanger L, Ivers H. The insomnia severity index: psychometric indicators to detect insomnia cases and evaluate treatment response. Sleep. 2011; 34(5): 601-8.

28. Miller MW, Wolf EJ, Reardon AF, Harrington KM, Ryabchenko K, Castillo D, et al. PTSD and conflict behavior between veterans and their intimate Partners. J Anxiety Disord. 2013; 27(2): 240-51.

29. Kleim B, Ehlers A, Glucksman E. Investigating cognitive pathways to psychopathology: predicting depression and posttraumatic stress disorder from early responses after assault. Psychol Trauma. 2012; 4(5): 527-37.

30. Kip KE, Sullivan KL, Lengacher CA, Rosenzweig L, Hernandez DF, Kadel R, et al. Brief treatment of symptoms of post-traumatic stress disorder (PTSD) by use of accelerated resolution therapy (ART). Front Psychiatry. 2013; 4(8): 11. doi: 10.3389/ fpsyt.2013.00011. 